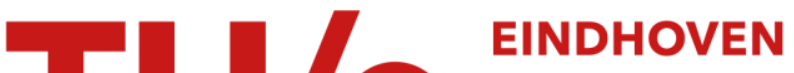 UNIVERSITY OF TECHNOLOGY
}

\section{Frequency response data based optimal control using the data based symmetric root locus}

\section{Citation for published version (APA):}

Hoogendijk, R., Hamer, den, A. J., Angelis, G. Z., Molengraft, van de, M. J. G., \& Steinbuch, M. (2010). Frequency response data based optimal control using the data based symmetric root locus. In 2010 IEEE International Conference on Control Applications part of 2010 IEEE Multi-conference on Systems and Control, Yokohama, Japan, September 8-10, 2010 (pp. 257-262). Institute of Electrical and Electronics Engineers. https://doi.org/10.1109/CCA.2010.5611199

DOI:

10.1109/CCA.2010.5611199

Document status and date:

Published: 01/01/2010

\section{Document Version:}

Publisher's PDF, also known as Version of Record (includes final page, issue and volume numbers)

\section{Please check the document version of this publication:}

- A submitted manuscript is the version of the article upon submission and before peer-review. There can be important differences between the submitted version and the official published version of record. People interested in the research are advised to contact the author for the final version of the publication, or visit the $\mathrm{DOI}$ to the publisher's website.

- The final author version and the galley proof are versions of the publication after peer review.

- The final published version features the final layout of the paper including the volume, issue and page numbers.

Link to publication

\section{General rights}

Copyright and moral rights for the publications made accessible in the public portal are retained by the authors and/or other copyright owners and it is a condition of accessing publications that users recognise and abide by the legal requirements associated with these rights.

- Users may download and print one copy of any publication from the public portal for the purpose of private study or research.

- You may not further distribute the material or use it for any profit-making activity or commercial gain

- You may freely distribute the URL identifying the publication in the public portal.

If the publication is distributed under the terms of Article 25fa of the Dutch Copyright Act, indicated by the "Taverne" license above, please follow below link for the End User Agreement:

www.tue.nl/taverne

Take down policy

If you believe that this document breaches copyright please contact us at:

openaccess@tue.nl

providing details and we will investigate your claim. 


\title{
Frequency Response Data Based Optimal Control using the Data Based Symmetric Root Locus
}

\author{
Rob Hoogendijk, ${ }^{1}$ Arjen den Hamer, ${ }^{2}$ Georgo Angelis, ${ }^{3}$ René van de Molengraft ${ }^{4}$ and Maarten Steinbuch ${ }^{5}$ \\ 1,2,4,5 Department of Mechanical Engineering \\ Eindhoven University of Technology \\ P.O. Box 513, 5600 MB Eindhoven, The Netherlands \\ ${ }^{1}$ r.hoogendijketue.nl, ${ }^{2}$ a.j.d.hamerdtue.nl, \\ ${ }^{4} \mathrm{~m} \cdot j \cdot g \cdot \mathrm{v} \cdot \mathrm{d} \cdot \mathrm{molengraft}$ etue.nl, \\ ${ }^{5}$ m. steinbuch@tue.nl
}

\begin{abstract}
This paper describes a data-based frequency domain optimal control synthesis method. Plant frequency response data is used to compute the frequency response of the controller using a spectral decomposition of the optimal return difference. The underlying cost function is selected from a databased symmetric root-locus, which gives insight in the closedloop pole locations that will be achieved by the controller. A simulation study shows the abilities of the proposed method.
\end{abstract}

\section{INTRODUCTION}

Since many motion systems are designed to have linear, time-invariant behavior, they can be characterized very well by their frequency response behavior. For such systems, large amounts of input-output data can be obtained at low cost. As a result, the majority of industrial controllers for mechatronic systems is designed using data-based controller synthesis methods. In this approach, frequency response data (FRD) is used to loop-shape the controller. The disadvantage of loopshaping is that it gives only limited insight in properties such as pole locations and it can not give guarantees about the optimality of the resulting controller in the sense of closedloop norm specifications.

Model-based control design approaches on the other hand can provide information on the mentioned properties, but deriving a low-order, accurate model for control design is not a straightforward task. Therefore, it would be convenient to have a controller synthesis method that can combine the best of both worlds: to have a controller synthesis method that gives insight in pole-locations and optimality of the controller, while using only frequency response data of the system to be controlled.

In most modern optimal control literature, state-space models are used to compute the controller. The linear quadratic regulator (LQR) is widely used, often in combination with the symmetric root locus (SRL) to acquire information on the relation between the cost function and the closed-loop pole locations [6]. However, these methods cannot be used directly when only FRD is available. Optimal control methods that use a model in the form of a transfer function also exist, see for example the Wiener-Hopf approach [1][2]. This method uses a spectral factorization of the optimal return difference to compute the controller.
Applications of this technique as described in [3], [4], [5] are all model based.

Existing data-based optimal control techniques, e.g. [7], [8], [9], [10], are all based on time-domain input-output data. Such techniques do not give insight in the closed-loop pole locations.

This paper describes frequency domain data-based optimal control. The proposed method gives the FRD of the controller that is optimal for a quadratic cost function by computing the spectral factorization of the optimal return difference, using only the FRD of the system to be controlled. For lightly damped systems, the SRL can be computed databased, which provides insight in the closed-loop pole locations beforehand without the use of a parametric model. The selection of the cost function, the computation of the controller and the controller structure are discussed. The realization of the controller from the computed FRD of the controller is beyond the scope of this paper. Summarizing, the main contributions of this paper are:

- A method to compute the FRD of the optimal controller using only FRD of the system to be controlled

- Data-based SRL to select the cost function based on the closed-loop poles that will be achieved by the controller

The paper is organized as follows. First the optimal control problem is formulated. Next, it is explained how the cost function can be selected based on the desired closed-loop pole locations of the system. Then, the computation of the frequency response function of the controller is discussed, and the structure of the resulting controller is explained. Finally, an example of the application of the proposed methods on a simulated mass-spring-damper system is presented.

\section{PROBLEM FORMULATION}

Let $H(s)$ be a stable single-input-single-output (SISO) system for which

$$
Y(s)=H(s) U(s),
$$

where $Y(s)$ is the output of the system and $U(s)$ the input. The goal of the output feedback controller $C(s)$, i.e. $U(s)=$ $C(s) Y(s)$, is to minimize the cost function

$$
J=\int_{0}^{\infty} \rho y(t)^{2}+u(t)^{2} d t,
$$


where $\rho$ is a scalar that determines the relative importance of the output $u(t)$ versus the performance variable $y(t)$ in the cost function. The choice of a suitable cost function, i.e. a suitable value for $\rho$ is crucial for the performance of the controller.

Suppose that only frequency response data (FRD) $H\left(j \omega_{i}\right)$ of the system to be controlled is available. The subscript $i$ is used to emphasize that discrete data-points are considered. Then, the problem can be formulated as follows:

Problem Statement. Given the frequency response data $H\left(j \omega_{i}\right)$ of a stable system, find the frequency response data of the optimal controller $C\left(j \omega_{i}\right)$ that minimizes (2), without the use of a parametric model.

Two main steps will be discussed to solve this problem:

1) Select a suitable value for $\rho$ (Sec. III)

2) Compute the controller $C\left(j \omega_{i}\right)$ (Sec. IV)

\section{COST FUNCTION FROM SRL}

It is well known that the optimal pole locations that minimize (2) lie at the stable roots of the symmetric root locus (SRL) equation [11],

$$
1+\rho H(-s) H(s)=0 .
$$

To compute solutions of this equation, the transfer function $H(s)$ should be known. Since the goal is to compute the controller without a model using only FRD, a different approach has to be followed. FRD only gives information about the value of the transfer function on the imaginary axis, i.e. $H\left(j \omega_{i}\right)$. The data that is needed to evaluate the SRL equation is called transfer function data (TFD), $H\left(s_{i}\right)$, which gives the value of the transfer function at points $s_{i}$ in the complex plane. Recently, it was shown in [12], [13] that Cauchy's integral formula can be used to compute the transfer function at points $j \omega_{i}$ that do not lie in the original set of measured responses $H\left(j \omega_{i}\right)$ in order to assess the inter frequency grid behavior of a system. Here, it will be shown that a similar technique can be used to compute TFD points $H\left(s_{i}\right)$ in the complex plane rather than on the imaginary axis as was done in [13]. The following two sections discuss how to compute TFD and how to use it in the SRL equation to select the $\rho$ from the cost criterion.

\section{A. Computing Transfer Function Data from Frequency Re- sponse Data}

Computing TFD from FRD is performed in two steps. First, a Cauchy integral [14] is used to compute all the points $H\left(s_{i}\right)$ in the right half plane (RHP) from the FRD $H\left(j \omega_{i}\right)$. The Cauchy integral formula is stated in the following theorem:

Theorem 1. (Cauchy integral formula) Let $f(z)$ be an analytical function in a region $G, \mathcal{C}$ a simple closed (Jordan) curve in $G$ and let a denote a point in the $\mathcal{C}$. Then

$$
\frac{1}{2 \pi j} \int_{\mathcal{C}} \frac{f(z)}{z-a} d z=f(a)
$$

Proof: For the proof, see [14].

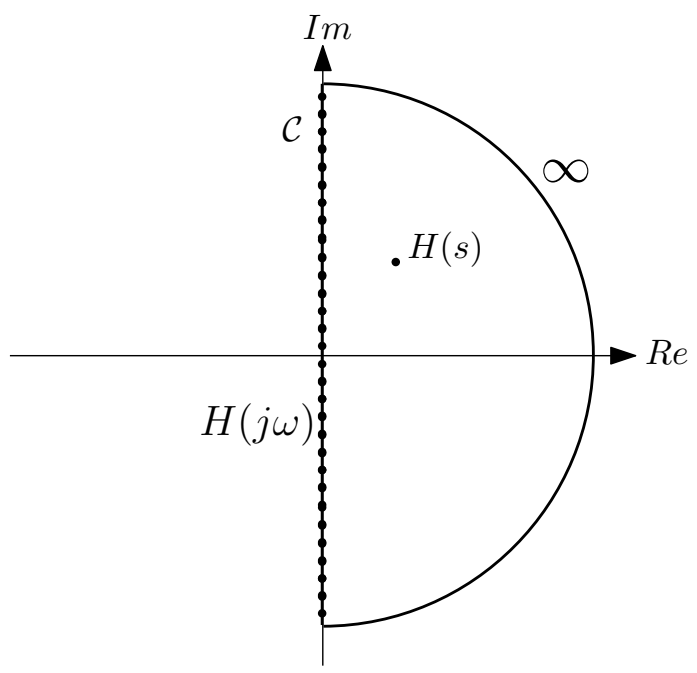

Fig. 1: Computation of $H(s)$ from $H(j \omega)$ by evaluation of a contour integral.

Fig. 1 illustrates how $H\left(s_{i}\right)$ is computed using a D-contour integral over all points $H\left(j \omega_{i}\right)$. For stable systems there are no poles in the right half plane (RHP), which makes the RHP analytic such that the Cauchy integral formula holds. Furthermore, assume that the response of the system at infinite frequency is zero. Then only the part of the Dcontour that lies on the imaginary axis contributes to the contour integral. This simplifies the Cauchy integral to

$$
H(s)=\frac{1}{2 \pi} \int_{-\infty}^{\infty} \frac{H(j \omega)}{(j \omega-s)} d \omega,
$$

which can be approximated by

$$
H\left(s_{i}\right)=\frac{1}{2 \pi} \sum_{k} \frac{H\left(j \omega_{k}\right)}{\left(j \omega_{k}-s_{i}\right)} \Delta \omega .
$$

This shows that for the RHP it is possible to compute the value of the transfer function $H\left(s_{i}\right)$ using only the FRD $H\left(j \omega_{i}\right)$.

The second step is to compute TFD for the LHP. Since the poles of the system lie in the LPH, it is a non analytic region for which (4) does not hold. Therefore a different technique is required to compute $H\left(s_{i}\right)$ in the LPH. It is assumed that the system is lightly damped. Therefore, the poles of the system will lie close to the imaginary axis which causes $H(s)$ to be symmetric in the imaginary axis. This makes it possible to approximate the LHP from the RHP with the relation

$$
H(s) \approx H(-s) .
$$

Thus, for lightly damped systems it is possible to compute TFD from FRD for the whole complex plane.

\section{B. Selection of $\rho$ from the TFD based SRL}

The symmetric root locus (SRL) can aid in the selection of the cost criterion parameter $\rho$ in (2), because it gives insight in the closed-loop pole locations. TFD can be used 
to compute the SRL of a system in a data-based way by rewriting (3)

$$
\begin{aligned}
1+\rho H\left(-s_{i}\right) H\left(s_{i}\right) & =0 \\
H\left(-s_{i}\right) H\left(s_{i}\right) & =-\frac{1}{\rho} \\
H\left(s_{i}\right) H\left(s_{i}\right) & =-\frac{1}{\rho},
\end{aligned}
$$

where (7) is used in the last step. Hence, the SRL can be computed from TFD by searching for points in $H\left(s_{i}\right) H\left(s_{i}\right)$ that have phase $-180^{\circ}$, i.e. $-\rho$ is a negative real number. With the SRL, it is possible to select the desired closedloop poles locations by selecting a point on the SRL. The corresponding value of $\rho$ can be computed from this point on the SRL and this value will be used in the cost function (2).

\section{DATA-BASED OPTIMAL CONTROL}

\section{A. Computation of the controller}

The goal is to compute the FRD $C\left(j \omega_{i}\right)$ of a controller $C(s)$ that minimizes the cost function (2). Using simple manipulation of the algebraic Riccati equation

$$
P A_{s s}+A_{s s}^{T} P-P B_{s s} B_{s s}^{T} P+\rho C_{s s}^{T} C_{s s}=0,
$$

where $A_{s s}, B_{s s}, C_{s s}$ denote the matrices of the state-space description of the system, it can be shown [15] that

$$
T(-s) T(s)=\rho H(-s) H(s)+1,
$$

with

$$
T(s)=1+C(s) H(s) .
$$

$T(s)$ is called the return difference, which is optimal if it satisfies (12). By computing a spectral factorization of the right hand side of the optimal return difference, it is possible to compute $C(s)$. First, the definition of spectral factorization is given.

Definition 2. (Spectral factorization) [1] Let $Z(s)$ denote a transfer function. Suppose $Z(s)$ can be written as

$$
Z(s)=Y(s) Y(-s) \text {. }
$$

Let $Y(s)$ be a transfer function that has all its poles and zeros in the LHP and consequently, $Y(-s)$ is the same function but it has all its poles and zeros in the RHP, which are the mirror images of the poles and zeros of $Y(s)$. Then

$$
\begin{aligned}
& (Z(s))^{+}=Y(s) \\
& (Z(s))^{-}=Y(-s) .
\end{aligned}
$$

In the remainder of this paper the shorthand notations $\bar{Y}:=Y(-s)$ and $Y:=Y(s)$ will be used frequently, in order to be able to omit the dependency on $s$, which clarifies the derivations. Using Definition 2 the controller can be computed, as stated in the following theorem.

Theorem 3. The controller that minimizes the cost criterion of (2) is given by

$$
C(s)=\left((\rho \bar{H} H+1)^{+}-1\right) H^{-1}
$$

Proof: Since it is required that $T(s)$ leads to a stable, minimum-phase closed-loop system, it must have all its poles and zeros in the LHP. Therefore, $T(s)$ can be derived from a spectral factorization of the optimal return difference. Since

$$
\begin{aligned}
\bar{T} T & =\rho \bar{H} H+1 \\
& =(\rho \bar{H} H+1)^{-}(\rho \bar{H} H+1)^{+},
\end{aligned}
$$

it must hold that

$$
T=(\rho \bar{H} H+1)^{+} .
$$

Using (13), the controller can be computed from

$$
\begin{aligned}
C(s) & =(T-1) H^{-1} \\
& =\left((\rho \bar{H} H+1)^{+}-1\right) H^{-1} .
\end{aligned}
$$

The difficulty of computing (17) lies in the computation of the stable spectral factor $(\rho \bar{H} H+1)^{+}$from data only. Fortunately, the magnitude of the spectral factor can be computed easily. Recall that the spectral decomposition separates the LHP and RHP terms of the transfer function. The function

$$
(1+\rho H \bar{H})=(1+\rho H \bar{H})^{+}(1+\rho H \bar{H})^{-}
$$

is the product of both spectral factors, which can be computed from the FRD directly. The amplitude of $(\rho \bar{H} H+1)^{+}$ can be computed from this function. Since the amplitude of the LHP factor is equal to the amplitude of the RHP factor (it is a symmetric function), the amplitude of (22) is the square of $(\rho \bar{H} H+1)^{+}$. Thus

$$
\left|(1+\rho H \bar{H})^{+}\right|=\sqrt{|(1+\rho H \bar{H})|}
$$

gives the amplitude of the spectral factorization. The phase of this stable spectral factor can be computed from this amplitude, using the Bode gain-phase relationship, assuming that the closed-loop system is minimum-phase. There are several ways to implement this relation, see for example [16].

Since the controller description (17) only depends on $H(s)$ and $\rho$, the frequency response of the controller $C\left(j \omega_{i}\right)$ can be computed directly from

$$
\begin{aligned}
& C\left(j \omega_{i}\right)= \\
& \left(\left(\rho H\left(-j \omega_{i}\right) H\left(j \omega_{i}\right)+1\right)^{+}-1\right) H\left(j \omega_{i}\right)^{-1} .
\end{aligned}
$$

using only the FRD $H\left(j \omega_{i}\right)$ of the system, and the choice of a suitable $\rho$, using the SRL as discussed in Section III.

\section{B. Controller structure explained}

The controller described by (17) can be regarded as the combination of a state observer and a state feedback controller. Equation (17) will be rewritten for the purpose of this interpretation. The transfer function of a system $H(s)$ can be written as

$$
H(s)=\frac{q(s)}{p(s)}=\frac{\sum_{i=0}^{m} q_{i} s^{i}}{\sum_{i=0}^{n} p_{i} s^{i}}, \quad m<n
$$

where $q(s)$ and $p(s)$ denote the numerator and denominator polynomial of $H(s)$. With the spectral factor

$$
\gamma(s)=(p \bar{p}+\rho q \bar{q})^{+}
$$




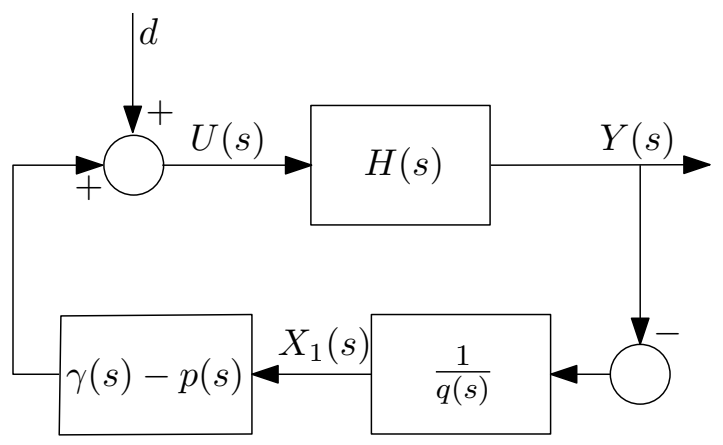

Fig. 2: The controller can be divided in two parts.

it is possible to rewrite the optimal controller as

$$
\begin{aligned}
C(s) & =\left((\rho \bar{H} H+1)^{+}-1\right) H^{-1} \\
& =\left(\left(\frac{\bar{p} p+\rho \bar{q} q}{\bar{p} p}\right)^{+}-1\right) \frac{p}{q} \\
& =\left(\frac{\gamma}{p}-1\right) \frac{p}{q} \\
& =\frac{\gamma(s)-p(s)}{q(s)} .
\end{aligned}
$$

Fig. 2 visualizes this division of the optimal controller in two parts.

Proposition 4. The $\frac{1}{q(s)}$ part of (30) is an observer for the states of the system $H(s)$.

Proof: The system $H(s)$ can be transformed using a similarity transformation to controllable canonical form

$$
\begin{aligned}
A & =\left[\begin{array}{cccc}
0 & 1 & & \\
& \ddots & \ddots & \\
& & 0 & 1 \\
-p_{0} & \cdots & -p_{n-2} & -p_{n-1}
\end{array}\right] \\
B & =\left[\begin{array}{llll}
0 & \cdots & 0 & 1
\end{array}\right]^{T} \\
C & =\left[\begin{array}{lll}
q_{0} & \cdots & q_{n-1}
\end{array}\right]
\end{aligned}
$$

The states $X_{1}=\left[\begin{array}{llll}x_{1} & x_{2} & \cdots & x_{n}\end{array}\right]^{T}$ of this representation satisfy

$$
\begin{aligned}
x_{2} & =\frac{d}{d t} x_{1} \\
x_{3} & =\frac{d}{d t} x_{2}=\frac{d^{2}}{d t^{2}} x_{1} \\
& \vdots \\
x_{n} & =\frac{d}{d t} x_{n-1}=\frac{d^{n-1}}{d t^{n-1}} x_{1}
\end{aligned}
$$

Thus all states can be represented as derivatives of the first state $x_{1}$. The output $y(t)$ is computed from

$$
\begin{aligned}
y(t) & =C x(t) \\
& =\left[\begin{array}{llll}
q_{0} x_{1} & q_{1} x_{2} & \cdots & q_{n-1} x_{n}
\end{array}\right] \\
& =\left[\begin{array}{llll}
q_{0} & q_{1} \frac{d}{d t} & \cdots & q_{n-1} \frac{d^{n-1}}{d t^{n-1}}
\end{array}\right] x_{1},
\end{aligned}
$$

which uses the special structure of the states of the canonical representation. Transforming to the Laplace domain yields

$$
\begin{aligned}
Y(s) & =\left[\begin{array}{llll}
q_{0} & q_{1} s & \cdots & q_{n-1} s^{n-1}
\end{array}\right] X_{1}(s) \\
& =q(s) X_{1}(s)
\end{aligned}
$$

Therefore the state $X_{1}(s)$ can be computed from

$$
X_{1}(s)=\frac{Y(s)}{q(s)}
$$

which shows that the $\frac{1}{q(s)}$ part of (30) is an observer for the state $X_{1}(s)$ of the system $H(s)$. Because of the canonical form, the information of all states is contained in $X_{1}(s)$.

Before the state feedback part $\gamma(s)-p(s)$ can be described, the properties of $\gamma(s)$ are described in the following lemma.

Lemma 5. Let $H(s)$ denote a transfer function which can be written in terms of its numerator and denominator polynomial, $H(s)=q(s) / p(s)$. Consider the controller $C=(\gamma(s)-p(s)) / q(s)$, where $\gamma(s)=(p \bar{p}+\rho q \bar{q})^{+}$. In this case it holds that:

1) $\gamma(s)$ is the closed-loop characteristic polynomial

2) The roots of $\gamma(s)$ are the optimal closed-loop pole locations given the cost function (2).

Proof:

1) Substitution of the controller $C(s)$ and system $H(s)$ in the denominator of the closed-loop transfer function gives

$$
\frac{1}{1+C H}=\frac{1}{1+\frac{\gamma-p}{q} \frac{q}{p}}=\frac{p}{\gamma},
$$

which shows that $\gamma(s)$ is the closed-loop characteristic polynomial.

2) The optimal closed-loop poles for (2) satisfy the symmetric root locus equation [6]

$$
(1+\rho \bar{H} H)^{+}=0
$$

The zeros of $\gamma(s)$ are equal to the solutions of the symmetric root locus equation. This can be seen by considering

$$
(1+\rho \bar{H} H)^{+}=\frac{(p \bar{p}+\rho q \bar{q})^{+}}{(p \bar{p})^{+}}=\frac{\gamma}{p}
$$

Proposition 6. The $\gamma(s)-p(s)$ part of the optimal controller (30) places the closed-loop poles at the locations that are optimal for cost criterion (2)

Proof: From Lemma 5 it is clear that $\gamma(s)$ is the desired closed-loop characteristic polynomial that is optimal in terms of the cost criterion (2). This is exactly the closedloop polynomial that the state feedback controller $\gamma(s)-p(s)$ achieves, when it is applied to the state $X_{1}(s)$. Utilizing the special structure of the states in canonical form of (31) again gives

$$
\begin{aligned}
u(s) & =[\gamma(s)-p(s)] X_{1}(s) \\
& =\left[\left(\gamma_{0}-p_{0}\right)+\left(\gamma_{1}-p_{1}\right) s+\ldots\right] X_{1}(s) \\
& =k_{0} x_{1}+k_{1} x_{2}+\ldots+k_{n-1} x_{n}
\end{aligned}
$$

This is equivalent to a standard pole placement algorithm as found in [6] for example. In such a pole placement algorithm, 
the gains for each state are equal to the difference between the coefficients of the characteristic equation of the system $p_{i}$ and the coefficients of the desired closed-loop characteristic equation $\gamma_{i}$, thus in this case

$$
k_{i}=\gamma_{i}-p_{i} .
$$

which completes the proof.

Summarizing, the optimal controller (24) can be interpreted as an observer that estimates $X_{1}(s)$ with information on all states of the system, combined with a state feedback controller that places the poles at the optimal locations, given by the roots of $\gamma(s)$.

\section{Simulation Study}

This section gives an example of the application of the developed controller synthesis method on a mass-springdamper system, see Fig. 3. A collocated controller will be designed to control the position of the first mass. FRD $H\left(j \omega_{i}\right)$ is generated from a theoretical model for this system using the force on the first mass as input and the position of the first mass as output, thus

$$
H\left(j \omega_{i}\right)=\frac{x_{1}}{F_{1}} .
$$

First the SRL will be drawn. Therefore, the TFD $H\left(s_{i}\right)$ is computed from this FRD data using the Cauchy integral (6) and the symmetry condition (7). Using the TFD in (10) gives the SRL of which one quadrant is shown in Fig. 4. The other quadrants are similar to this one, but mirrored in the real and imaginary axes.

The SRL shows the open-loop pole locations $(\mathbf{x})$, zero locations (o), the computed SRL points (.) and the SRL computed from the analytical model (-). The data-based SRL matches the analytical model quite good, except for points close to the imaginary axis, where the symmetry condition is not valid.

The selection of the value of $\rho$ will be based on the lower branch for this example. Each location on this branch corresponds to a different choice of $\rho$. To show the influence of the selection of the parameter $\rho$ in the cost criterion on the resulting controller, three different choices for $\rho$ will be selected $(\nabla, \circ, \square)$. Since the points are selected based on the SRL data, the actual pole locations ( $\mathrm{x}$ ) will differ slightly from the selected locations. The $\rho$ value shown by the $\circ$ is the optimal point in terms of damping in the system.

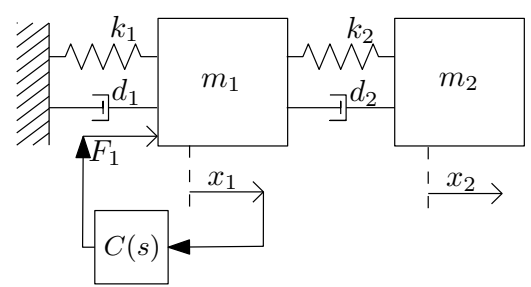

Fig. 3: Fourth order system consisting of two masses connected via springs and dampers.

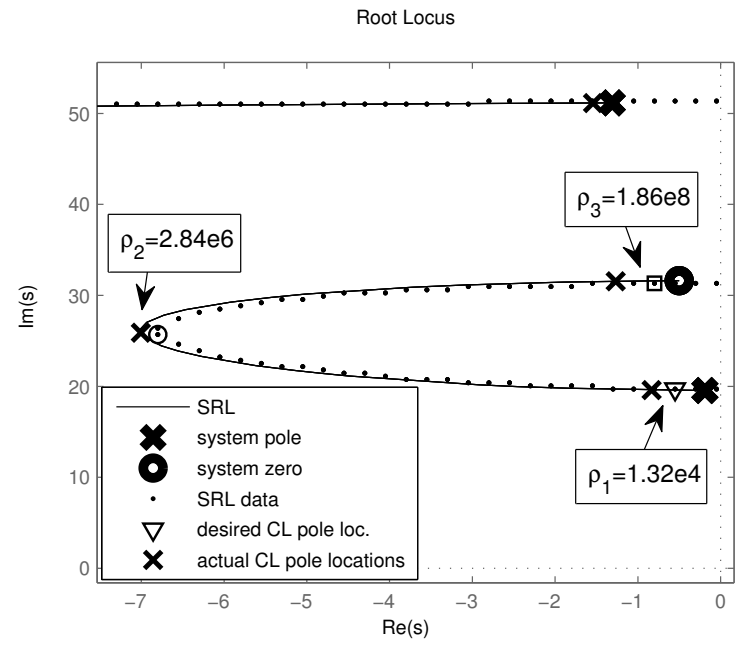

Fig. 4: SRL computed from FRD (.) and analytic solution (-) showing three choices for $\rho: \nabla, \circ, \square$ and the corresponding closed-loop pole locations computed from the model: $(\mathbf{x})$.
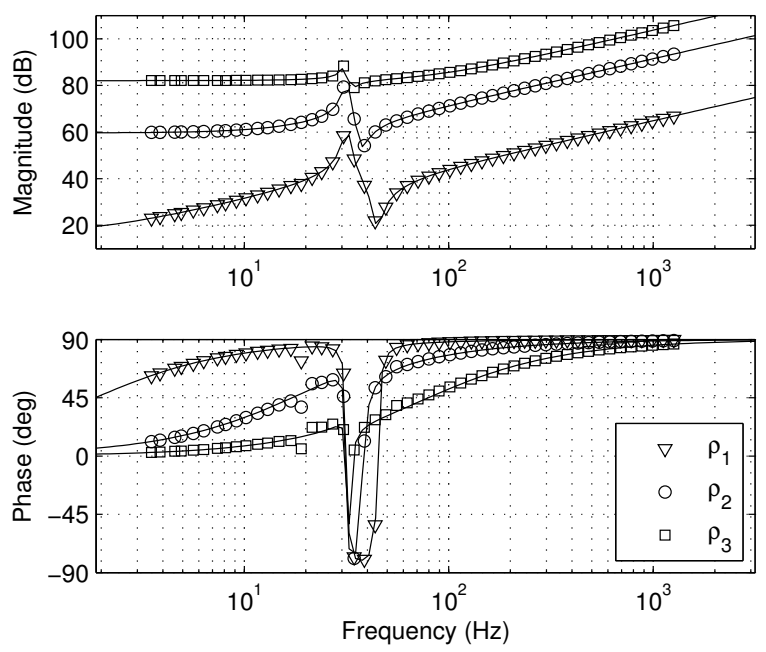

Fig. 5: Bode diagram of the controller for three $\rho$ values, and analytic solution (-).

Using these values of $\rho$ and the FRD, the resulting controllers can be computed using (24), see Fig. 5. The results from the data-based method are plotted with $\nabla, \circ, \square$, while the analytic solutions are shown by the solid lines. Again, there is a good match between the data-based and analytic solution.

Suppose that the goal of the controller is to suppress force disturbances $d$ that enter the loop before the plant, see Fig. 2. The relevant transfer function from the disturbance $d$ to the output $y$ is the process sensitivity given by

$$
P S=\frac{H}{1+C H} .
$$



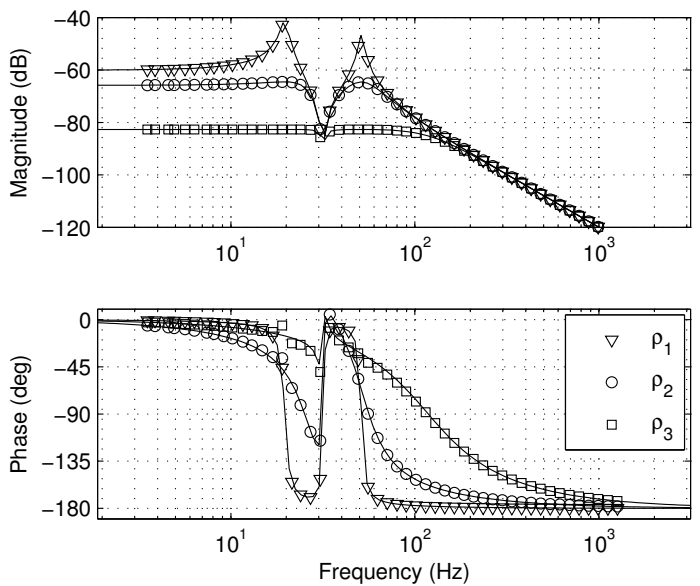

Fig. 6: Bode diagram of the process sensitivity for three $\rho$ values, and analytic solution (-).
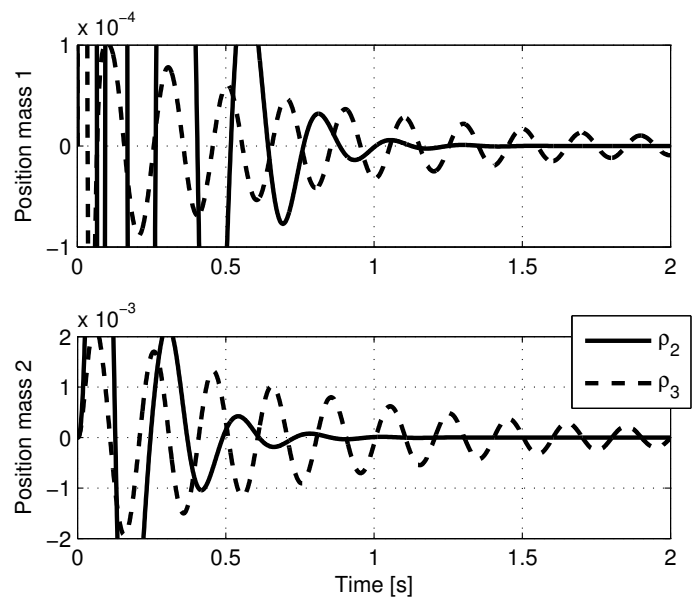

Fig. 7: Impulse response from $d$ to output for $\rho$ with maximum damping (-) and for high gain case $(--)$. The response corresponding to $\rho_{1}$ is omitted for clarity.

This transfer function is shown in Fig. 6, both computed from the FRD and the model.

The process sensitivity seems to suggest that $\rho_{3}$ would be the best for disturbance rejection. However, the impulse response shown in Fig. 7 clearly shows a better response for $\rho_{2}$. Although initially the amplitude of the response for $\rho_{2}$ is higher, which agrees with the process sensitivity of Fig. 6 , the damping of the response is much better. This could be expected since the SRL shows that for this choice, the damping in the system is optimal. This shows the advantage of the insight that the SRL gives in the closed-loop pole locations of the system.

\section{CONCLUSIONS}

In this paper it has been shown that an optimal controller can be computed from data only, using the spectral factorization of the return difference of the system. This controller is based on a quadratic optimality criterion, of which the parameter $\rho$ is selected from a data-based symmetric root locus. The SRL gives information on the closed-loop pole locations of the system, which gives a fast quality assessment of the resulting controller. The advantage of being able to select the closed-loop poles beforehand has been shown in a simulation, where this technique is used to maximize the amount of damping in a system.

Concluding, it can be stated that an optimal controller can be computed fully data-based, and also the closed-loop pole locations can be selected data-based, using the method described in this paper. The method can only be applied to systems that have low damping, because of the assumed symmetry with respect to the imaginary axis. However, since many high tech motion systems satisfy this property, the approach will be feasible in practice.

\section{REFERENCES}

[1] M. Grimble and M. Johnson, Optimal Control and Stochastic Estimation. Wiley Interscience, 1988.

[2] J. H. Davis, "A distributed filter derivation without riccati equations," in Decision and Control including the 17th Symposium on Adaptive Processes, 1978 IEEE Conference on, vol. 17, Jan. 1978, pp. 127-129.

[3] B. Willis and R. Brockett, "The frequency domain solution of regulator problems," Automatic Control, IEEE Transactions on, vol. 10, no. 3, pp. 262-267, Jul 1965.

[4] B. Avramovic, N. Barkakati, and G. L. Blackenship, "Application of a spectral factorization approach to the control of flexible structures," in Decision and Control, 1984. The 23rd IEEE Conference on, vol. 23, Dec. 1984 , pp. 1695-1696.

[5] C.-M. Lin and T.-S. Lu, "On the frequency domain linear quadratic optimal systems design," in Decision and Control, 1994., Proceedings of the 33rd IEEE Conference on, vol. 3, Dec 1994, pp. 2609-2614 vol.3.

[6] G. F. Franklin, J. Powell, and A. Emami-Naeini, Feedback control of dynamical systems. Prentice-Hall, 2002.

[7] R. Skelton and G. Shi, "The data-based lqg control problem," in Decision and Control, 1994., Proceedings of the 33rd IEEE Conference on, vol. 2, Dec 1994, pp. 1447-1452 vol.2.

[8] K. Nordstrom, E. Karlsson, and A. Malmgren, "On the data based time varying lqg controllers via cholesky factorisations," in Decision and Control, 1995., Proceedings of the 34th IEEE Conference on, vol. 4, Dec 1995, pp. 3414-3419 vol.4.

[9] W. Aangenent, D. Kostic, B. de Jager, R. v. d. Molengraft, and M. Steinbuch, "Data-based optimal control," in American Control Conference, 2005. Proceedings of the 2005, June 2005, pp. 1460-1465 vol. 2 .

[10] J. Dong and M. Verhaegen, "Model free probabilistic design with uncertain markov parameters identified in closed loop," in Decision and Control, 2008. CDC 2008. 47th IEEE Conference on, Dec. 2008, pp. 2637-2643.

[11] T. Kailath, Linear systems. Englewood Cliffs, 1980.

[12] A. den Hamer, S. Weiland, M. Steinbuch, and G. Angelis, "Stability and causality constraints on frequency response coefficients applied for non-parametric h2 and h8 control synthesis," in Decision and Control, 2008. CDC 2008. 47th IEEE Conference on, Dec. 2008, pp. 3670 3675.

[13] A. den Hamer, S. Weiland, and M. Steinbuch, "Worst-case inter frequency grid behavior of transfer functions identified via finite frequency response data," in Proc. of European Control Conference 2009, Budapest, 2009, p. 446.

[14] M. Rahman, Complex variables and transform calculus. Computational Mechanics Publications, 1997.

[15] A. Macfarlane, "Return difference and return ratio matrices and their use in analysis and design of multivariable feedback control systems," in $I E E$, vol. 117, no. 10, 1970, p. 2037.

[16] L. Buzan, C. Rusu, R. Bilcu, and P. Kuosmanen, "Phase approximation in linear and logarithmic frequency domain," in Control, Communications and Signal Processing, 2004. First International Symposium on, 2004, pp. 709-712. 